\title{
Two-Phase Simulation of Wave-Induced Tunnel Scour beneath
}

\section{Marine Pipelines}

Abbas Yeganeh-Bakhtiary ${ }^{1,3, *}$, Mohammad Hossein Kazeminezhad ${ }^{2}$, Amir EtemadShahidi $^{3}$, Jaco H. Baas ${ }^{4}$

${ }^{1}$ Hydro-environmental Research Centre, School of Engineering, Cardiff University, Cardiff, CF24 3AA, Wales, United Kingdom

${ }^{2}$ School of Civil Engineering, Iran University of Science \& Technology, Tehran 16844, Iran

${ }^{3}$ Griffith School of Engineering, Gold Coast campus, Griffith University, Queensland, 4222

${ }^{4}$ School of Ocean Sciences, Bangor University, Menai Bridge, Anglesey LL59 5AB, United Kingdom

\begin{abstract}
An Eulerian two-phase flow model was presented and employed to investigate waveinduced tunnel scour beneath a marine pipelines. The model is based on the Euler-Euler coupled governing equations for the fluid and sediment phases, i.e. time-averaged continuity and momentum equations were solved for both phases with a modified $k-\varepsilon$ turbulence closure for the fluid phase. Fluid-particle, particle-particle and fluid-structure interactions were implemented in the simulation. The model accounts for the interphase momentum exchange by considering the drag, lift and added mass forces. The flow model was validated against an oscillatory flow around an isolated cylinder and a cylinder close to a rigid wall. The two-phase model was also validated against an
\end{abstract}

\footnotetext{
*Corresponding author, email address: abbasyeganeh@gmail.com
} 
oscillatory sheet-flow motion above a plane bed. Then, the two-phase model was used to simulate the wave-induced tunnel scour beneath the pipeline laid on a plane erodible bed. Comparison between the numerical results and experimental measurements indicates that the model simulates the bed profile successfully during the tunnel scour stage. Investigations revealed that the tremendous sediment transport takes place during the tunnel scour stage under high turbulence intensity. A phase-lag was observed between the flow velocity in the scour hole and the free stream velocity.

Keywords: Euler-Euler coupling; fluid-particle interaction; $k-\varepsilon$ turbulence model; oscillatory motion; particle-particle interaction; tunnel erosion scour; two-phase flow model 


\section{INTRODUCTION}

Marine pipelines are widely used to transport products from one location to another, but these pipelines are often put onto the seabed without any protection. In deep water regions, orbital wave motion has a minor effect on the near-bed pipelines. By contrast, in shallow water, the pipelines are affected severely by the wave-induced oscillatory motion. The wave-pipe interaction brings severe changes in the flow pattern around pipe, and it increases the local bed shear stress. Therefore, the pipeline may face local scouring if it is positioned onto an erodible seabed. This scouring is therefore controlled by the simultaneous interaction of the fluid flow, the pipe and the erodible seabed.

Generally, the scour beneath marine pipelines occurs in the onset, tunnel and lee-wake erosion stages (Mao 1986). The tunnel stage of scour beneath pipelines is highly complex and important from both applied engineering and sediment transport points of view. During this stage, substantial sediment transport takes place in a very short period of time and the main part of the scour hole just below the pipe is generated (Sumer and Fredsøe 2002). If intensive tunnel erosion occurs beneath the pipeline, use of an appropriate protective method is inevitable. The tunnel erosion stage, which succeeds the generation of a small gap between the pipe and seabed is more complicated in oscillatory flow than in unidirectional flow.

In the past decades, several experimental studies on wave-induced scour beneath pipelines have been carried out (e.g. Mao 1986; Sumer and Fredsøe 1990; Mousavi et al. 2009; Xu et al. 2010). Sumer and Fredsøe (1990) pointed out that the equilibrium scour depth for live bed conditions is governed by Keulegan-Carpenter number, $K C$, defined as: 
$K C=\frac{u_{m} T}{D}$

where $u_{m}$ is the maximum value of the undisturbed orbital velocity of water particles at the bed, $D$ is the pipe diameter and $T$ is the wave period or oscillatory flow period. Experimental results revealed that the scour depth increases as the $K C$ number increases.

In addition to the experimental studies, several efforts have been made to simulate the scour phenomenon beneath pipelines. The capability of numerical models to predict the local scour around pipelines relies on the accurate simulation of sediment transport. Two kinds of numerical models have been used to simulate the sediment transport process: single-phase models and two-phase models. In the single-phase models, it is assumed that the presence of sediment particles has no direct effect on the flow field (Greimann and Holly Jr 2001) and the sediment transport is determined using equilibrium or non-equilibrium sediment transport models (Wu 2007). In the two-phase models, the momentum equations are solved for both fluid and sediment phases considering inter-phase interactions. Recently, the sophisticated Euler-Euler coupling two-phase flow models became popular in simulation of sediment transport process. In these models, the fluid and sediment phases are treated as the separate continuous mediums. Therefore, the dynamics of the fluid and sediment phases, as well as the fluidparticle and particle-particle interactions, are considered in the simulations. Most of the two-phase Euler-Euler coupling models are based on the continuity and momentum equations for both fluid and sediment phases (Kobayashi and Seo 1985). In the early Euler-Euler models, simplified assumptions were made to facilitate the solution of the governing equations (Asano 1990; Ono et al. 1996). Dong and Zhang (1999) proposed a complete Euler-Euler coupling two-phase model for simulation of sediment transport at 
the sheet-flow regime in oscillatory flow motion. Recent Euler-Euler coupling studies were based on Dong and Zhang's (1999) two-phase model with some modifications (Liu and Sato 2006; Li et al. 2008; Bakhtyar et al. 2010). Although the Euler-Euler coupling approach has been widely used in one-dimensional sediment transport modeling, it has received little attention in simulating two-dimensional problems, such as scour around pipelines.

In the past three decades, several numerical models have been developed to simulate current-induced scour around marine pipeline. These models were based on the potential flow theory (Chiew 1991; Li and Cheng 1999) and turbulent flow theory (Brørs 1999; Liang et al. 2005a; Liang et al. 2005b). It was recognized that the potential flow theory is not capable of predicting the scour profile at the downstream side of pipe due to restrictions in simulating wake flow and vortex shedding. Moreover, the standard $k-\varepsilon$ model was found to be appropriate for simulation of current-induced scour around pipelines (Liang and Cheng 2005b; Liang et al. 2005a). So far, the numerical studies have focused mainly on simulation of scour under unidirectional flow condition. Only one numerical simulation of wave-induced scour around pipeline has been carried out (Liang and Cheng 2005a). In Liang and Cheng (2005a), waves were modeled as sinusoidal oscillatory flows and the flow field was determined by solving the ReynoldsAveraged Navier-Stokes (RANS) equations with a $k-\omega$ turbulence closure. Both bed load and suspended load were considered in the scour model. The time-dependent bed profile was obtained based on the period-averaged transport rate. The results showed favorable agreement between the predicted and measured bed profiles.

All previously mentioned studies on the numerical simulation of scour beneath pipelines were based on the single-phase models. Published examples of the application 
of Euler-Euler coupling two-phase model in the simulation of scour around pipelines are rare. Zhao and Fernando (2007) employed an Eulerian two-phase model embedded in FLUENT software to simulate the steady current-induced scour around pipelines. Although the simulated bed profiles were in good agreement with the measured bed profiles, Zhao and Fernando (2007) found that the model overestimates the sediment phase velocity below the water and sediment interface. Yeganeh-Bakhtiary et al. (2011) also presented an Euler-Euler coupling two-phase model to investigate the currentinduced tunnel erosion beneath submarine pipelines.

Our literature review reveals that the wave-induced tunnel erosion underneath the pipeline has not yet been investigated in detail. In the previous numerical studies, a small sinusoidal perturbation with amplitude $0.1 D$ was introduced to the initial bed profile. Therefore, the early stage of the scour, i.e. tunnel scour stage, was never simulated completely, and the simulated scour pit might be a function of the forced initial bed profile. The main aim of the study presented herein was to investigate the wave-induced tunnel scour beneath pipelines numerically. Since, tremendous sediment transport takes place in a short period of time in tunnel erosion stage, the fluid-particle and particle-particle interactions should be all considered in the simulation process. Moreover, due to the rapid changes in the bed profile, the model should be able to take into account the continuous changes in the bed profile and, in turn, simultaneous variations of the flow field. For this purpose, a two-dimensional Euler-Euler coupling model is presented, in which two different series of RANS equations are solved for the fluid and sediment phases. One of the novel features is the use of two-phase flow theory in the simulation of the wave-induced scour around pipelines. First, the flow model was evaluated against measured data including the lift force acting on the pipe, flow velocity 
rate in the gap between pipe and solid bed, and the velocity profile around an isolated cylinder. The two-phase model was also validated with experimental data on oscillatory sheet-flow conditions above a plane bed. Finally, the validated two-phase model was used to simulate the early stages of wave-induced scour around pipelines. The experimental data of Sumer and Fredsøe (1990) was used to evaluate the capability of the model to simulate the tunnel erosion. Unlike the previous studies, the numerical simulation was carried out by assuming an initial flat bed profile under the pipe. The scour pit shape, gap flow and sediment transport dynamics were investigated during the tunnel erosion stage.

\section{NUMERICAL MODEL}

In the present study, the numerical model of Yeganeh-Bakhtiary et al. (2011) was extended for oscillatory flow conditions. Despite the similarities between the fundamental approaches and models in both studies, the following essential differences can be highlighted:

- In the present model, correlations between fluctuating velocity and concentration were considered in the fluid and sediment phases momentum equations.

- In the present model the drag, lift and added mass forces were considered as the interaction forces between the fluid and sediment phases.

- The applied drag coefficient, boundary conditions and computational domain in the present model are different from those applied by Yeganeh-Bakhtiary et al. (2011).

\section{Governing Equations}


The hydrodynamics of the fluid phase were simulated using the 2D ReynoldsAveraged-Navier-Stokes (RANS) equations with a $k$ - $\varepsilon$ turbulence closure model. The sediment phase was embedded into the model by solving the time-averaged momentum and continuity equations. The governing equations were originally adopted from the Kobayashi and Seo (1985), and extensively employed in the literature (e.g., Dong and Zhang 1999; Li et al. 2008; Yeganeh-Bakhtiary et al. 2011). The continuity equations for the fluid and sediment phases, respectively, are:

$$
\begin{aligned}
& \frac{\partial\left(\rho_{f}(1-c)\right)}{\partial t}+\frac{\partial\left(\rho_{f}(1-c) u_{j}\right)}{\partial x_{j}}=\frac{\partial\left(\rho_{f} \overline{c^{\prime} u_{j}^{\prime}}\right)}{\partial x_{j}} \\
& \frac{\partial\left(\rho_{s} c\right)}{\partial t}+\frac{\partial\left(\rho_{s} c u_{s j}\right)}{\partial x_{j}}=-\frac{\partial\left(\rho_{s} \overline{c^{\prime} u_{s j}^{\prime}}\right)}{\partial x_{j}}
\end{aligned}
$$

The momentum equations for the fluid and sediment phases, respectively, are:

$$
\begin{aligned}
& \frac{\partial\left(\rho_{f}(1-c) u_{i}\right)}{\partial t}+\frac{\partial\left(\rho_{f}(1-c) u_{i} u_{j}\right)}{\partial x_{j}}=-\rho_{f}(1-c) g \delta_{i 2}-(1-c) \frac{\partial p}{\partial x_{i}}+\frac{\partial \tau_{i j}^{f}}{\partial x_{j}}-f_{i} \\
& \frac{\partial\left(\rho_{s} c u_{s i}\right)}{\partial t}+\frac{\partial\left(\rho_{s} c u_{s i} u_{s j}\right)}{\partial x_{j}}=-\rho_{s} c g \delta_{i 2}-c \frac{\partial p}{\partial x_{i}}+\frac{\partial \gamma_{i j}^{s}}{\partial x_{j}}+\frac{\partial \tau_{i j}^{s}}{\partial x_{j}}+f_{i}
\end{aligned}
$$

The fluid phase and sediment phase stresses, respectively, are:

$$
\begin{aligned}
& \tau_{i j}^{f}=\rho_{f} v(1-c)\left(\frac{\partial u_{i}}{\partial x_{j}}+\frac{\partial u_{j}}{\partial x_{i}}\right)-\rho_{f}(1-c) \overline{u_{i}^{\prime} u_{j}^{\prime}}+\rho_{f} u_{i} \overline{c^{\prime} u_{j}^{\prime}}+\rho_{f} u_{j} \overline{c^{\prime} u_{i}^{\prime}} \\
& \tau_{i j}^{s}=-\rho_{s} c \overline{u_{s i}^{\prime} u_{s j}^{\prime}}-\rho_{s} u_{s i} \overline{c^{\prime} u_{s j}^{\prime}}-\rho_{s} u_{s j} \overline{c^{\prime} u_{s i}^{\prime}}
\end{aligned}
$$


where $x_{i}(i=1,2)$ denotes the Cartesian coordinate system, the $x_{1}$ axis is taken in the horizontal direction $(x)$ and the $x_{2}$ axis in the vertical direction $(y)$. In the above equations, $t$ is time, $c$ is the volumetric sediment concentration, $u_{j}$ and $u_{s j}$ are the $x_{j}$ components of fluid and sediment velocities, $p$ is the pressure, $\rho_{f}$ and $\rho_{s}$ are the fluid and sediment densities, $g$ is the gravitational acceleration, $\delta_{i j}$ is the Kronecker delta, $\tau_{i j}^{f}$ is the viscous and turbulent stress tensor of the fluid phase, $\tau_{i j}^{s}$ is the stress tensor of the sediment phase, $\gamma_{i j}^{s}$ is the intergranular stress tensor, $f_{i}$ is the $x_{i}$ component of the interaction force per unit volume between fluid and sediment phases, and $v$ is the kinematic viscosity of fluid. All variables are time-averaged and the over-bar and prime denote the time correlations and the fluctuation quantities, respectively.

The main unknowns in the above equations are the fluid and sediment velocity vectors, pressure, and sediment concentration. Additional unknowns appear due to the time-averaging process, and consequently the unknowns outnumber the equations. Correlations between the fluctuating velocity components (Reynolds stresses) were modeled based on the Boussinesq assumption, while correlations between fluctuating velocity and concentration were modeled using a diffusive scheme (Kobayashi and Seo 1985; Longo 2005):

$$
-\overline{u_{i}^{\prime} u_{j}^{\prime}}=v_{t}\left(\frac{\partial u_{i}}{\partial x_{j}}+\frac{\partial u_{j}}{\partial x_{i}}\right)-\frac{2}{3} k \delta_{i j} \quad, \quad \overline{c^{\prime} u_{i}^{\prime}}=\overline{c^{\prime} u_{s i}^{\prime}}=-\frac{v_{t}}{\sigma_{c}} \frac{\partial c}{\partial x_{i}}
$$

where $v_{t}$ is the eddy viscosity, $k$ is the turbulent kinetic energy and $\sigma_{c}$ is the turbulent Schmidt's number for concentration. 
In the present study, the turbulence closure model should be able to estimate the turbulence field around the horizontal pipe as well as in the near-bed region at low to high sediment concentrations. In the two-phase flow modeling, motion of the sediment particles might be strongly affected by the fluid turbulence, and the presence of moving particles in the fluid field may lead to an extra source of production or dissipation of fluid turbulence. Therefore, the modified $k$ - $\varepsilon$ model, proposed by Elghobashi and AbouArab (1983) for two-phase flows, was used:. Details of the applied turbulence model have been presented in Yeganeh-Bakhtiary et al.'s (2011) study.

\section{Intergranular Stress and Interaction Forces}

The intergranular stresses were adopted according to Li et al. (2008) and Dong and Zhang (1999) as:

$$
\begin{aligned}
& \gamma_{x y}^{s}=1.2 \lambda^{2} \rho_{f} v \frac{\partial u_{s}}{\partial y} \\
& \gamma_{y y}^{s}=\left|\gamma_{x y}^{s}\right| \cot \phi
\end{aligned}
$$

where $\lambda=\left[\left(c_{m} / c\right)^{1 / 3}-1\right]^{-1}$ is the linear sediment concentration, $\phi$ is the friction angle, $c_{m}$ is the theoretical maximum sediment concentration and $u_{s}$ is the horizontal component of the sediment velocity. Equation (9) was originally proposed by Ahilan and Sleath (1987) and was successfully applied by Yeganeh-Bakhtiary et al. (2011) in the simulation of current-induced scour around pipelines. 
The interaction forces between the phases arise from the velocity difference between the fluid phase and the sediment phase. The major contributors to the interaction forces include the drag, lift and added mass forces, which were determined following Li and Sawamoto (1995) and Longo (2005):

$$
\begin{aligned}
& f_{x}=\frac{3}{4 d} \rho_{f} c C_{D} u_{r} \sqrt{u_{r}^{2}+v_{r}^{2}}-\rho_{f} c C_{M}\left(\frac{\partial u_{s}}{\partial t}-\frac{\partial u}{\partial t}\right) \\
& f_{y}=\frac{3}{4 d} \rho_{f} c C_{D} v_{r} \sqrt{u_{r}^{2}+v_{r}^{2}}+\frac{3}{4} \rho_{f} c C_{L}\left|u_{r}\right| \frac{\partial u_{r}}{\partial y}-\rho_{f} c C_{M}\left(\frac{\partial v_{s}}{\partial t}-\frac{\partial v}{\partial t}\right)
\end{aligned}
$$

where $u_{r}=u-u_{s}$ and $v_{r}=v-v_{s}$ are the relative velocities between the fluid and sediment, and $C_{D}, C_{M}$ and $C_{L}$ denote the drag, added mass and lift coefficients, respectively. $u$ and $u_{s}$ are the horizontal components of the fluid and sediment velocities, and $v$ and $v_{s}$ are the vertical components of the fluid and sediment velocities, respectively. The lift and added mass coefficients were set as 4/3 and 1/2, respectively (Li et al. 2008). The drag coefficient was estimated with the following equation (Maude and Whitmore 1958; Longo 2005):

$$
C_{D}=\left(\frac{24 v_{f}}{d \sqrt{u_{r}^{2}+v_{r}^{2}}}+2\right)(1-c)^{-4.5}
$$

\section{Computational Domain and Boundary Conditions}

The wave-induced scour around a horizontal pipe was simulated using a rectangular computational domain with a length of $40 D$ and a height of $10 D$ (Fig. 1). At the initial stage, a packed sand layer with maximum volumetric sediment concentration, $c_{0}$, was considered as the sediment phase at the bottom of the flow domain. The pipe center was located over the sand layer surface, $20 D$ from the lateral boundaries. A very small gap 
equal to one computational cell height was imposed between the base of the pipe and the bed surface because of numerical requirements. The applied gap herein is very small compared to that of the previous studies (e.g. Liang and Cheng 2005a) and does not influence the subsequent scouring process. At the inflow boundary, the velocity components and turbulence quantities varied periodically with time as follows (Liang and Cheng 2005a):

$$
\begin{array}{lc}
u(t)=u_{m} \sin \left(\frac{2 \pi t}{T}\right) \quad, \quad v(t)=0 \\
k(t)=k_{m}\left(\sin \left(\frac{2 \pi t}{T}\right)\right)^{2}, & \varepsilon(t)=\varepsilon_{m}\left|\sin \left(\frac{2 \pi t}{T}\right)\right|^{3}
\end{array}
$$

where $k_{m}=0.0005 u_{m}^{2}$ is the amplitude of the turbulent kinetic energy, and $\varepsilon_{m}=C_{\mu} k_{m}^{2} /(100 v)$ is the amplitude of the turbulent dissipation rate (Liang et al. 2005c). An et al. (2011) have also applied the above boundary conditions in their simulation of oscillatory flow around a cylinder above a plane bed.

At the free surface, the symmetric boundary conditions were applied (YeganehBakhtiary et al. 2011). The hydraulically smooth wall boundary condition was applied on the pipe surface similar to that applied by Kazeminezhad et al. (2010) and Liang and Cheng (2005b). Since sediment particles can be eroded and deposited, the bed profile changes with time and these changes needs to be tracked. Following Zhao and Fernando (2007), the bed profile was selected as the location for which the sediment concentration equals 0.5 . The rough wall boundary condition was applied for the bed surface. The sediment phase velocity components were set to zero at the rough wall boundary. The boundary conditions for $k$ and $\varepsilon$ at the bottom were based on the local equilibrium of 
turbulence energy near the bottom, accounting for the influence of the presence of sediment grains (Longo 2005; Bakhtyar et al. 2010).

\section{Numerical Method}

Eight partial differential equations (PDE) and several algebraic equations describe the fluid and sediment phases. The PDEs consist of two momentum equations for the fluid phase, two momentum equations for the sediment phase, two mass conservation equations respectively for the fluid and sediment phases, and two turbulence closure equations. The governing equations were discretized by the cell-centered Finite Volume Method (FVM) in a Cartesian coordinate system. Discretizations were carried out on a rectangular staggered mesh as illustrated in Fig. 2. The scalar variables (e.g., pressure and fluid and sediment concentrations) were stored at the nodes marked $(\bullet)$, while the velocities were defined at the scalar cell faces in between the nodes. In Fig. 2, horizontal arrows $(\rightarrow)$ indicate the locations for horizontal fluid and sediment phase velocities and vertical arrows $(\uparrow)$ denote those for vertical fluid and sediment phase velocities.

To discretize the governing equations, the conservation equations (Eqs. 2-5), were presented using following general phasic equation:

$$
\frac{\partial\left(\rho_{t} c_{t} \phi_{t}\right)}{\partial t}+\nabla \cdot\left(\rho_{t} c_{t} \mathbf{u}_{t} \phi_{t}\right)=\nabla \cdot\left(c_{t} \Gamma_{t} \nabla \phi_{t}\right)+S_{t}
$$

where $\mathbf{u}$ is the velocity vector and subscribe $t$ denotes the fluid or sediment phase. The expression for $\Gamma_{t}$ and $S_{t}$ can be deduced from the parent equations. Integrating the general conservation equation over a control volume and using the Gauss' divergence theorem to transform the volume integral into a surface integral yields: 


$$
\int_{C V} \frac{\partial\left(\rho_{t} c_{t} \phi_{t}\right)}{\partial t} d V+\int_{A} \mathbf{n} .\left(\rho_{t} c_{t} \mathrm{u}_{t} \phi_{t}\right) d A=\int_{A} \mathbf{n} .\left(c_{t} \Gamma_{t} \nabla \phi_{t}\right) d A+\int_{C V} S_{t} d V
$$

where $V$ is the volume of the control volume, $A$ is the area of the control volume faces and vector $\mathbf{n}$ is normal to the surface area $d A$. By replacing the surface integrals with a summation of the fluxes over the side of the control volumes, Eq. (17) transforms to:

$$
\frac{\partial\left(\rho_{t} c_{t} \phi_{t} V\right)}{\partial t}+\sum_{N B=e, w, n, s}\left(\mathbf{J}_{t_{N B}}^{C}\right)=\sum_{N B=e, w, n, s}\left(\mathbf{J}_{t_{N B}}^{D}\right)+S_{t} V
$$

where $\mathbf{J}_{t_{N B}}^{C}$ and $\mathbf{J}_{t_{N B}}^{D}$ are the convective and diffusive fluxes, respectively. The fluxes were discretized using a hybrid scheme that combines upwind and central differencing schemes based on the cell Peclet number (Hiltunen et al. 2009).

The solution procedure of the discretized PDEs has been explained in YeganehBakhtiary et al. (2011). The flow fields close to the pipe and the bed surface are complicated due to the presence of shear layers and high vertical gradients of sediment concentration. The mesh size in the vertical direction $(\Delta y)$ in the bed vicinity was similar to the median sediment diameter, i.e., for the test cases with $d_{50}=0.58 \mathrm{~mm}$ and $d_{50}=0.18 \mathrm{~mm}, \Delta y$ was set to $0.5 \mathrm{~mm}$ and $0.25 \mathrm{~mm}$, respectively. The computational domain close to the pipe was discretized using 75 grid points per pipe diameter in horizontal and vertical directions. The mesh size was gradually increased to reach its maximum value near the lateral and free surface boundaries. Because of the time-scale differences in sediment and flow processes, different time marching were adopted for the sediment and fluid phase calculations. For the fluid phase simulation, the time step was set to $0.002 \mathrm{~s}$, whereas, the time step for simulation of the sediment phase was 
selected as $0.0001 \mathrm{~s}$. The numerical tests carried out indicated that the selected time steps are small enough to ensure that the solutions are time-step independent.

\section{MODEL VALIDATION}

\section{Validation of the Fluid Phase Model}

Numerical simulation of wave-induced scour relies on accurate simulation of flow pattern around a near-bed cylinder. The present fluid phase model has been validated for steady flows around a near-bed cylinder (Kazeminezhad et al. 2010). Here, it is validated against available experimental data of oscillatory flow around a circular cylinder.

First, the fluid phase model was validated against the measured velocity profile around an isolated cylinder at $K C=5$ and $R e=100$ (Dütsch et al. 1998). The experimental data set of Dütsch et al. (1998) has been used to validate several numerical models (e.g., Choi et al. 2007; An et al. 2011). The experiment was carried out in a stationary tank with fluid at rest, in which a 1-cm diameter cylinder was oscillated with a purely sinusoidal motion. The experimental conditions were simulated as an oscillatory flow around an isolated cylinder with $u_{m}=1 \mathrm{~cm} / \mathrm{s}$ and $T=5 \mathrm{~s}$. The simulated horizontal and vertical velocity profiles at four sections at phase position $\theta=180^{\circ}$ were compared with the measured profiles in Fig. 3. The numerical results agree reasonably well with the experimental data. However, some differences were found between the predicted and measured velocity profiles at some sections. Similar discrepancies were observed in other numerical models (Choi et al. 2007; An et al. 2011).

Second, the model's ability to simulate the oscillatory flow velocity below the near-wall cylinder was investigated. If a circular cylinder is placed near a plane bed 
with a small gap, high-speed jet flow prevails in the gap. Jarno-Druaux et al. (1995) measured the flow velocity around a near-wall pipe under wave motion in a wave flume. These experiments were carried with a pipe diameter of $0.02 \mathrm{~m}$ and for two different gap to diameter ratios $(e / D)$ of 0.09 and 0.5 . The wave height, wave period and corresponding $K C$ and $R e$ numbers were $0.047 \mathrm{~m}, 1.08$ s, 4.9 and 1800, respectively. The flow velocity rate in the gap was calculated based on the horizontal component of flow velocity, $u(t)$, measured over the downstream tangential profile of the cylinder as follows:

$q_{u}=\frac{\int_{y=0}^{y=e+D / 2} u(t) d y}{e+D / 2}$

The experiments of Jarno-Druaux et al. (1995) were simulated as an oscillatory flow with a maximum undisturbed velocity of $0.091 \mathrm{~m} / \mathrm{s}$ and wave period of $1.08 \mathrm{~s}$. Figure 4 compares the measured and simulated flow velocity rates in the gap during the oscillatory flow motion. The model simulates the gap flow quite well for both values of $e / D$, with a small under-prediction of the maximum value of $q_{u}$. For $e / D=0.09$, the gap between the pipe and bed was smaller than the thickness of the wave boundary layer over the plane bed. Therefore, the gap flow was strongly influenced by the wave boundary layer. Figure 4 clearly shows the expected phase-lag between the flow velocity in the gap and free stream velocity, especially for $e / D=0.09$. The obtained phase-lag is about $41^{\circ}$, which confirms that flow reversal occurred earlier in the gap than in the upper part of the flow, which is in good agreement with the observations. To investigate whether the flow phase-lag in the gap was equal to that of the wave boundary layer over a plane bed, the numerical simulation was also carried out in the absence of the pipe. The results show a phase-lag between the free stream velocity and 
the flow velocity close to the boundary of about $33^{\circ}$. It thus appears that the presence of the pipe close to the bed leads to the generation of an extra phase-lag between the free stream velocity and the velocity at the bed-side of pipe. For $e / D=0.5$, the phase-lag between the flow velocity in the gap and the velocity of the free flow was not as large as for $e / D=0.09$. This may be attributed to the weaker effect of the wave boundary layer on the gap flow in the latter case. Figure 4 shows that in both cases the numerical simulations underestimated the peak values of the flow velocity in the gap. This may be explained by a slight difference between the wave-induced flow around the pipe in the experiment and the pure oscillatory flow assumed in the simulation.

Finally, the fluid phase model was validated against measured hydrodynamic forces acting on a near-wall cylinder. Time variation of the lift force acting on the pipe records the vortex motion and vortex shedding phenomena around the pipe. Here, the experimental conditions of Sumer et al. (1991), with $D=9 \mathrm{~cm}, e / D=0.4$ and $K C=20$ were simulated. Figure 5 shows the comparison of the simulated and measured lift coefficients. The lift coefficient was defined as $C_{L}=F_{L} /\left(0.5 \rho D U^{2}{ }_{\mathrm{m}}\right)$, where $F_{L}$ is the force component in the vertical direction. The results depict a small phase-lag and some differences between the measured and simulated lift coefficient. This may be originated because of the slight difference between the numerical and experimental conditions. For example, the experimental condition, in which the cylinder was oscillated in still water, was simulated as a purely oscillatory flow motion. However, the numerical results are in reasonably good agreement with the experimental data of Sumer et al. (1991). The numerical model was particularly successful in the simulation of time variation of the lift coefficient as well as its amplitude. Since the variation of the lift coefficient during oscillatory flow is strongly related to the vortex dynamics around the cylinder, it can be 
concluded that the fluid phase model was capable of predicting vortex motion around circular cylinder.

\section{Validation of the Two-Phase Model}

The two-phase model has been validated against simulation of steady sheet-flow and steady current-induced tunnel scour below a pipe (Yeganeh-Bakhtiary et al. 2011). Here, the two-phase model is validated against oscillatory sheet-flow motion above a plane bed using the experimental data of Horikawa et al. (1982), who performed the sheet-flow experiment in an oscillatory flow tunnel for sand sediment and measured the concentration, sediment velocity and sediment flux at six different phases. The median sediment diameter, velocity amplitude and oscillation period were $0.2 \mathrm{~mm}, 1.27 \mathrm{~m} / \mathrm{s}$ and $3.6 \mathrm{~s}$, respectively.

The simulation was carried out using a 12 -cm computational domain with a 2$\mathrm{cm}$ thick sand layer covered by a 10-cm high pure fluid zone at the initial stage. Figure 6 illustrates the simulated and measured vertical profiles of the sediment concentration at six phase angles, and shows that the present model is capable of predicting the concentration distribution in the sheet-flow layer. The normalized concentration at initial bed level $(y=0)$ is about 0.5 at most phases. Close to this level, a high vertical concentration gradient was generated due to a high gradient in the sediment-water mixture density. Above this level at top layer of the sheet-flow layer, the concentration gradient becomes relatively low. To further investigate the numerical results, the predicted and measured normalized sediment flux profiles at different phases are compared in Fig. 7. Above the stable part of the bed, the sediment flux increased as the sediment velocity increased. The sediment flux achieved its maximum value around the 
initial bed level. Farther away from the bed, although the sediment velocity was relatively high, the sediment flux was negligible due to the low sediment concentration. Hence, most of the sediment transport took place at the thin bottom and middle part of the sheet-flow layer, where the sediment concentration and particle-particle interactions were most important. Generally, there is a good agreement between the measured and simulated sediment flux profiles in terms of profile shape and magnitude. The largest difference between measured and predicted sediment fluxes were found at around the initial bed level, where a high sediment concentration gradient existed. Similar differences have been observed in previous numerical studies (e.g., Liu and Sato 2006).

\section{SIMULATION OF TUNNEL SCOURING}

To verify the ability of the new two-phase model to simulate wave-induced scour, two experiments by Sumer and Fredsøe (1990) were simulated. These experiments were carried out in a 0.6-m wide wave flume with a piston-type wave generator. Table 1 lists the experimental conditions, including the wave and bed material parameters. In both cases, the hydraulically smooth pipe was placed over the sand bed without any initial gap and the bed profile was measured at different time instances. Sumer and Fredsøe (1990) found that the scour hole just below the pipe developed immediately by means of tunnel erosion. For example, for experiment 1 , the scour depth reached $S / D=0.2$ after 0.2 minute, while the equilibrium scour depth, $S / D=0.28$, was reached after 55 minutes. It is evident that in a short amount of time, after the passage of only a few waves over the pipe, the scour depth attained to $71 \%$ of its equilibrium depth. 
In the numerical simulation, the wave-induced flow was approximated by an ideal oscillating flow with velocity amplitudes and oscillation periods identical to those listed in Table 1. One of the novel features of the numerical simulation herein was the beginning of scour from an initial flat bed profile beneath the pipe, unlike previous models. Figure 8 a shows a good agreement between the simulated bed profile and the measured bed profile for experiment 1 of Sumer and Fredsøe (1990) at $t=0.2 \mathrm{~min}$. A small discrepancy exists between the simulated and measured bed profiles at the upstream side of the pipe, which is attributed to the approximation of the experimental wave-induced flows by means of pure oscillatory flows. The comparison between the simulated bed profile for experiment 2 at $t=1 \mathrm{~min}$ and experimental data of Sumer and Fredsøe (1990) is shown in Fig. 8b. The agreement between the computed and measured bed profiles is reasonably good. In experiment 2 , the mounds of scour-derived sediment were smoother than that in experiment 1 . This difference in mound shape is attributed to the larger $K C$ number and smaller sediment size for experiment 2, and thus higher concentrations of suspended sediment particles than in experiment 1 . Due to the limitations of the previous experimental and numerical models, details of the scour hole development, flow hydrodynamics and dynamics of sediment transport during the wave-induced tunnel erosion below pipeline have not carefully investigated. Therefore, in the next sections these issues will be discussed in more detail for experiment 1.

\section{Scour Development}

Figure 9 displays snapshots of the sediment concentration field at several time instances for experiment 1 , where the bed surface can be approximated by the top of the red 
region. After a half oscillation at $t=0.7 \mathrm{~s}$, shortly before the reversal of the oscillatory flow motion, a very small mound of sediment is generated at rear side of the pipe. After the first flow oscillation, two end mounds appear near the pipe, and as the scour hole develops, the mounds become larger and they move away from the pipe. It is clear that in a short amount of time, substantial volumes of sediment were removed from underneath the pipe, and a scour hole develops very rapidly. This was mainly caused by a strong jet flow within the scour hole, which is discussed below. Figure 10 shows that the rate of deepening of the scour progressively decreases with time.

\section{Flow Dynamics around the Pipe}

At the tunnel scour stage, a jet-like flow prevails in the gap, which plays an important role in the scour development underneath the pipeline. In the literature, the gap flow has been investigated numerically and experimentally for cases in which the pipe is exposed to a steady current (e.g., Smith and Foster 2005; Oner et al. 2008). However, the gap flow for cases where the pipe undergoes of oscillatory flow motion has not been analyzed yet.

Figure 11 depicts the gap flow velocity profile under the pipe $(x / D=0)$ at six phases during the 8th oscillatory motion. The horizontal velocity and vertical distance were normalized by the amplitude of the velocity at the free surface, $u_{m}$, and pipe diameter, $D$, respectively. In Fig. $11, y / D=0$ corresponds to the base of the pipe, while $y / D$, at which the horizontal velocity becomes zero, corresponds to the bed surface. As seen at most phases, the maximum gap flow velocity considerably exceeds the amplitude of the free surface velocity. For example, when $S / D$ is about 0.18 , the 
maximum velocity of the gap flow is $2.3 u_{m}$. At phase $\theta=0^{\circ}$, when the flow is reversed at the free surface and its velocity becomes zero, the gap flow velocity is rather high. This shows further evidence for a phase-lag between the flow at the free surface and below the pipe. The velocity profile at phase $\theta=150^{\circ}$ shows that close to the wall boundaries, i.e. near the pipe surface as well as near the bed surface, the horizontal fluid velocity becomes negative, even though it is still positive at the center of the gap flow. This implies that the flow reversal occurred earlier close to the wall boundaries. In fact, this new finding is related to a specific property of oscillatory boundary layers, in that the velocity near the boundary turns before the free stream velocity (Nielsen 1992).

It is also readily observed in Fig. 11 that there is a phase-lag between the free surface flow and the gap flow, as the maximum velocities of the free flow and the gap flow occur at $t / T=7.25$ and $t / T=7.15$, respectively. Therefore, during the 8 th oscillatory motion, when $S / D$ was about 0.18 , the phase-lag between the free surface velocity and gap flow velocity is about $35.5^{0}$. To further investigate this observation, the phase-lag was calculated during the scour hole development. Figure 12 shows the variation of the phase lag, $d \varphi$, with the nondimensional scour depth. At the early stage of scouring, when the scour depth is rather small, the phase-lag between the free surface velocity and the gap flow velocity is highest. This may be due to the strong influence of the oscillatory boundary layer on the gap flow (Jarno-Druaux et al. 1995). At the early stages, the phase-lag quickly decreases with increasing scour depth. For deeper scours, the phase-lag decreases at a progressively lower rate.

Figure 13 depicts the maximum velocity of the gap flow during each oscillatory motion against the nondimensional scour depth. The maximum velocity of the gap flow increases with increasing scour depth until a maximum value is reached at $S / D=0.18$. 
This is in line with the experimental results of Jarno-Druaux et al. (1995) for the small gap between the pipe surface and solid bed, where they argued that the restricted flow through the gap is a kind of the low energy boundary layer flow. Figure 13 also shows that the gap flow becomes slightly weaker for scour depths larger than $S / D=0.18$. Generally, it appeared that the maximum fluid velocity of the gap flow for oscillatory flow was higher than for steady currents (cf. Jensen et al. 1990; Oner et al. 2008). Sumer and Fredsøe (2002) also pointed out that the water discharge in the gap is much larger in oscillatory flow than in the steady currents, which confirms the new abovementioned numerical finding.

During the first half of the oscillation, the fluid flows from upstream to downstream (left to right). Therefore, vortices may develop at certain phases on rear side of pipe. Figure 14 shows the flow velocity field on the rear side of pipe at $\theta=30^{\circ}$ and $\theta=150^{\circ}$. During the flow acceleration at $\theta=30^{\circ}$, there was no vortex close to the bed, while during the flow deceleration at $\theta=150^{\circ}$, several vortices can be recognized close to the pipe and the bed surface. The generated vortices may produce extra turbulence close to the bed, which will be discussed later.

\section{Dynamics of Sediment Transport}

In previous numerical simulations, the sediment transport was considered in terms of bed load and suspended load. A number of bed load transport formulae have been proposed for steady, uniform current conditions, but the application of these formulae for oscillatory flows is not straightforward. Moreover, because of their empirical nature, different bed load transport formulae do not produce consistent results. One of the 
advantages of the Euler-Euler coupling approach applied herein is the ability to simulate the sediment transport without employing such empirical bed load transport formulae. Since the sediment transport mechanism during the early stages of the wave-induced scour has not yet been investigated, numerical findings on dynamics of sediment transport are presented.

Figure 15 plots the computed sediment transport rate under the pipe at $x / D=0$ during the tunnel scour stage. The transport rate was estimated by vertically integrating the sediment flux under the pipe, $Q_{s}=\int u_{s} c d z$. During the first oscillation, the sediment transport rate was rather small. In time, the sediment transport rate increased as the scour depth increased. The timing of the peak value of the sediment transport rate corresponded to the timing of maximum gap flow; thereafter, a slight decrease was predicted in the sediment transport rate. Note that some part of the transported sediment load deposited at the upstream and downstream sides of the pipe immediately the flow reversed, which leads to the generation of the two mounds on either side of the pipe.

In order to further investigate the sediment transport mechanism, Fig. 16 displays normalized sediment concentrations (left panel) and sediment flux profiles (right panel) under the pipe $(x / D=0)$ at six phases during the 8th oscillation. In the vertical direction, three regions with distinct features can be defined. In the bottom region, the sediment concentration is high during the entire oscillation. Due to the high sediment concentration and intense particle-particle interactions, the velocity of the fluid and sediment phases is relatively low in this region. The sediment flux is also low in the bottom region, but it becomes higher with increasing $y / D$. In the middle region, the sediment concentration decreases sharply with increasing vertical distance from the 
bottom. The large sediment concentration gradient corresponds to a large negative water and sediment mixture density gradient, which resulting in flow stratification (Liu and Sato 2006). Figure 11 showed that the fluid phase and sediment phase velocities were high in the middle region. This explains the peak value in the sediment flux profiles for this region (Fig. 16). In the top region, the sediment concentration and the sediment flux are low and variable in time. Figure 16 shows that the sediment flux under the pipe increases in the first half of the oscillation until it reached a maximum value at about $\theta$ $=60^{\circ}$, while it decreased afterwards.

The suspended sediment transport rate is directly related to the turbulence velocities. To investigate the probability of transport in the form of suspended sediment, the turbulence velocity, $\sqrt{u^{\prime 2}}$, normalized by particle settling velocity, $w_{s}$, at a distance of $3 \mathrm{~mm}$ above the bed surface for different phases is plotted in Fig. 17. The figure shows that the turbulence velocities under the pipe are higher than at the upstream and downstream sides of the pipe. This can be ascribed to turbulence production by the accelerating flow underneath the pipe. Moreover, the turbulence velocities were higher at downstream side of the pipe than at the upstream side of the pipe. The high turbulence velocities in this region appear to be generated by a vortex located close to the sediment bed, as was seen in Fig. 14. Figure 17 shows that the turbulence velocity under the pipe $(x / D=0)$ reaches its maximum value at $\theta=60^{\circ}$, when the maximum gap flow velocity occurs under the pipe. It should also be noted from Fig. 17 that during extended periods the turbulence velocities near the scour hole were equal to or greater than the particle settling velocity. As the turbulence velocity needs to exceed the settling velocity to render suspended sediment transport possible (Boothroyd 1971), it is therefore concluded that, according to the model, at least part of the sediment load was 
transported in suspension underneath the pipe. Locations of reduced turbulence intensity at either side of the pipe may have promoted the settling of sediment particles, and thus the formation of the mounds depicted in Fig. 9.

\section{CONCLUSIONS}

In this study, the wave-induced tunnel scour beneath a pipeline was investigated numerically based on Euler-Euler coupling two-phase flow theory. The continuity and momentum equations for both fluid and sediment phases were solved to quantify the dynamics of the fluid and sediment phases. The inter-phase momentum exchange was considered via the drag, lift and added mass forces. The fluid turbulence field was modeled based on the modified $k-\varepsilon$ turbulence closure. The fluid phase and two-phase models were first verified by simulating the oscillatory flow around a cylinder close to a rigid bed and by simulating oscillatory sheet-flow sediment transport, respectively. Then, the two-phase model was employed to simulate wave-induced tunnel scour. The simulated bed profiles compared well with the experimental measurements of Sumer and Fredsøe (1990).

The major outcomes of this study can be summarized as follows:

- The Euler-Euler coupling approach was capable of predicting the wave-induced scour profile and two ends mounds around the pipe. At the early stages, the scour depth rapidly increased with time, and thereafter the rate of increase of scour depth decreased in time. 
- The maximum velocity of the gap flow increased with increasing scour depth, until the velocity reached a maximum value. Thereafter, the maximum gap flow velocity decreased slightly with further increases of the scour depth.

- A phase-lag between the gap flow and free stream flow was found, and the phase-lag decreased as the scour depth increased.

- During the course of oscillatory motion, the turbulence velocity near the scour pit exceeded the sediment settling velocity. Consequently, the sediment particles can be transported in suspension.

- The sediment transport rate under the pipe increased rapidly at the early stage of the tunnel scour and then reduced gradually.

\section{ACKNOWLEDGMENTS}

The authors are grateful to the anonymous reviewers for their helpful comments. The writers would like to acknowledge the support from the Iran National Science Foundation. 


\section{References}

Ahilan, R.V., Sleath, J.F.A. (1987). "Sediment transport in oscillatory flow over flat bed.” Journal of Hydraulic Engineering, 113(3), 308-322.

An, H., Cheng, L., Zhao, M. (2011). "Steady streaming around a circular cylinder near a plane boundary due to oscillatory flow." Journal of Hydraulic Engineering, 137(1), 2333.

Asano, T. (1990). “Two-phase flow model on oscillatory sheet-flow." Proceeding, 22nd International Conference of Coastal Engineering, ASCE, 2372-2384.

Bakhtyar, R., Barry, D. A., Yeganeh-Bakhtiary, A., Li, L., Parlange, J. Y.,Sander, G. C. (2010). "Numerical simulation of two-phase flow for sediment transport in the innersurf and swash zones.” Advances in Water Resources, 33(3), 277-290.

Boothroyd, R. G. (1971). "Flowing gas-solids and suspensions." Chapman \& Hall, London.

Brørs, B. (1999). "Numerical modeling of flow and scour at pipelines." Journal of Hydraulic Engineering, 125(5), 511-522.

Chiew, Y. M. (1991). "Prediction of maximum scour depth at submarine pipelines." Journal of Hydraulic Engineering, 117(4), 452-466.

Choi, J. I., Oberoi, R. C., Edwards, J. R., Rosati, J. A. (2007). “An immersed boundary method for complex incompressible flows." Journal of Computational Physics, 224, $757-784$.

Dong, P., Zhang, K. (1999). "Two-phase flow modelling of sediment motions in oscillatory sheet-flow." Coastal Engineering, 36(2), 87-109. 
Dütsch, H., Durst, F., Becker, S., Lienhart, H. (1998). "Low- Reynolds-number flow around an oscillating circular cylinder at low Keulegan-Carpenter numbers." Journal of Fluid Mechanics, 360, 249-271.

Elghobashi, S. E., Abou-Arab, T. W. (1983). "A two-equation turbulence model for two-phase flows." Physics of Fluids, 26(4), 931-938.

Horikawa, K., Watanabe, A., Katori, S. (1982). "Sediment transport under sheet-flow condition." Proceeding, 18th International Conference of Coastal Engineering, ASCE, $1335-1352$.

Greimann, B.P., Holly Jr, F.M. (2001). "Two-Phase Flow Analysis of Concentration Profiles.” Journal of Hydraulic Engineering, 127(9), 753-762.

Hiltunen, K., Jäsberg, A., Kallio, S., Karema, H., Kataja, M., Koponen, A., Manninen, M., Taivassalo, V. (2009). "Multiphase Flow Dynamics: Theory and Numerics." Technical Research Centre of Finland (VTT).

Jarno-Druaux, A., Sakout, A., Lambert, E. (1995). "Interference Between a Circular Cylinder and a Plane Wall Under Waves.” Journal of Fluids and Structures, 9(2), 215230.

Jensen, B. L., Sumer, B. M., Jensen, H. R., Fredsoe, J. (1990). "Flow around and forces on a pipeline near a scoured bed in steady current." Journal of Offshore Mechanics and Arctic Engineering, 112(3), 206-213.

Kazeminezhad, M.H., Yeganeh-Bakhtiary, A., Etemad-Shahidi, A. (2010). "Numerical investigation of boundary layer effects on vortex shedding frequency and forces acting upon marine pipeline.” Applied Ocean Research, 32, 460-470.

Kobayashi, N., Seo, S. (1985). "Fluid and sediment interaction over a plane bed." Journal of Hydraulic Engineering, 111(6), 903-921. 
Li, F., Cheng, L. (1999). "Numerical model for local scour under offshore pipelines." Journal of Hydraulic Engineering, 125(4), 400-406.

Li, L., Sawamoto, M. (1995). "Multi-phase model on sediment transport in sheet-flow regime under oscillatory flow.” Coastal Engineering Journal, 38(2), 157-178.

Li, M., Pan, S., O'Connor, B. A. (2008). “A two-phase numerical model for sediment transport prediction under oscillatory sheet-flows." Coastal Engineering, 55(12), 11591173.

Liang, D., Cheng, L. (2005a). "Numerical model for wave-induced scour below a submarine pipeline." Journal of Waterway, Port, Coastal and Ocean Engineering, 131(5), 193-202.

Liang, D., Cheng, L. (2005b). "Numerical modeling of flow and scour below a pipeline in currents. Part I. Flow simulation.” Coastal Engineering, 52(1), 25-42.

Liang, D., Cheng, L., Li, F. (2005a). "Numerical modeling of flow and scour below a pipeline in currents: Part II. Scour simulation.” Coastal Engineering, 52(1), 43-62.

Liang, D., Cheng, L.,Yeow, K. (2005b). "Numerical study of the Reynolds-number dependence of two-dimensional scour beneath offshore pipelines in steady currents." Ocean Engineering, 32(13), 1590-1607.

Liang, D. F., Cheng, L.,Yeow, K. (2005c). "A numerical model for natural backfill of pipeline trenches subjected to unidirectional/oscillatory flows." China Ocean Engineering, 19(2), 269-286.

Liu, H., Sato, S. (2006). "A two-phase flow model for asymmetric sheetflow conditions." Coastal Engineering, 53(10), 825-843.

Longo, S. (2005). "Two-Phase Flow Modeling of Sediment Motion in Sheet-Flows above Plane Beds.” Journal of Hydraulic Engineering, 131(5), 366-379. 
Mao, Y. (1986). "The interaction between a pipeline and an erodible bed." $\mathrm{PhD}$ thesis, Institute of hydrodynamics and hydraulics engineering. Lyngby, Technical university of Denmark.

Maude, A. D., Whitmore, R. L. (1958). “A generalized theory of sedimentation.” British Journal of Applied Physics, 9(12), 477.

Mousavi, M. Yeganeh-Bakhtiary, A., Enshaei N. (2009). "The equivalent depth of wave-induced scour around offshore pipelines." Journal of Offshore Mechanics and Arctic Engineering, 131, 1-5.

Nielsen, P. (1992). “Coastal bottom boundary layers and sediment transport.” World Scientific, Singapore.

Oner, A. A., Salih Kirkgoz, M., Sami Akoz, M. (2008). "Interaction of a current with a circular cylinder near a rigid bed." Ocean Engineering, 35(14-15), 1492-1504.

Ono, M., Deguchi, I., Sawaragi, T. (1996). "Numerical modeling of sediment transport for various mode". Proceeding, 25th International Conference on Coastal Engineering, ASCE, 3888-3900.

Patankar, S.V., Spalding, D. B. (1972). "A calculation procedure for heat, mass and momentum transfer in three-dimensional parabolic flows." International Journal of Heat and Mass Transfer, 15, 1787.

Smith, H. D., Foster, D. L. (2005). "Modeling of flow around a cylinder over a scoured bed.” Journal of Waterway, Port, Coastal and Ocean Engineering, 131(1), 14-24.

Sumer, B. M., Fredsøe, J. (1990). "Scour below pipelines in waves." Journal of Waterway, Port, Coastal and Ocean Engineering, 116(3), 307-323.

Sumer, B. M., Fredsøe, J. (2007). "The mechanics of scour in the marine environment." World Scientific, Singapore (revised version). 
Sumer, B. M., Jensen, B. L., Fredsoe, J. (1991). "Effect of a plane boundary on oscillatory flow around a circular cylinder.” Journal of Fluid Mechanics, 225, 271-300. Wu, W. (2007). "Computational river dynamics", Taylor and Francis, UK. Xu, J., Li, G., Dong, P., Shi, J. (2010). "Bedform evolution around a submarine pipeline and its effects on wave-induced forces under regular waves." Ocean Engineering, 37(23), 304-313.

Yeganeh-Bakhtiary, A., Kazeminezhad, M.H., Etemad-Shaidi, A., Baas, J.H., Cheng, L. (2011). "Euler-Euler two-phase flow simulation of tunnel erosion beneath marine pipelines.” Applied Ocean Research, 33, 137-146.

Zhao, Z., Fernando, H. J. S. (2007). "Numerical simulation of scour around pipelines using an Euler-Euler coupled two-phase model." Environmental Fluid Mechanics, 7(2), 121-142. 